\title{
Missbrauch an katholischen Schulen
}

\author{
Peter Beer
}

Um es gleich vorneweg zu sagen: Eine historiographische Erforschung des Zusammenhangs des Phänomens Missbrauch mit dem System „katholische Schulen" stellt eine komplexe Aufgabenstellung dar. Grund dafür ist zum einen die notwendige Klärung dessen, was unter Missbrauch verstanden werden soll, will man sich nicht schon im Vorhinein „nur“ auf sexuellen Missbrauch und den von Erwachsenen gegenüber Minderjährigen beschränken. Zum anderen überlagern sich sowohl unterschiedliche gesellschaftliche, kirchliche und fachspezifische Entwicklungslinien als auch ganz verschiedene Ausgangspunkte, von denen aus das definiert wird, was unter einer katholischen Schule verstanden werden soll. Die katholische Schule als solche gibt es nicht. Eine Historiographie von Missbrauch an katholischen Schulen hat dies zu berücksichtigen, denn täte sie es nicht, wären Fehlschlüsse auf Grund mangelnder Differenzierung historiographischer Forschung bezüglich Ursachen und Zusammenhängen von Missbrauch und Eigenart bzw. Profil katholischer Schulen fast unvermeidlich.

$\mathrm{Zu}$ den Faktoren, die eine einheitliche historiographische Betrachtung katholischer Schulen mit dem Fokus Missbrauch erschweren, können u.a. gezählt werden: 1) die auf Grund des deutschen bundesrepublikanischen Föderalismus in Sachen Schule differierenden staatlichen Vorgaben, die kirchliche Schulen zu berücksichtigen haben, wollen sie staatlich anerkannt sein; 2 ) die unterschiedlichen gesellschaftlichen Funktionszuweisungen in Richtung kirchlicher Schulen in synchron wie diachron unterschiedlichen Staatsformen (Kaiserreich, Weimarer Republik, Nationalsozialismus, Sozialismus, bundesrepublikanische Demokratie) sowie die damit auf kirchlicher Seite verbundenen Isolationsmechanismen, Instrumentalisierungen etc.; 3) die verschiedenen Organisationsgrade und Funktionalitäten des kirchlichen Schulwesens entsprechend der jeweiligen Trägerschaft durch Orden, Diözesen, Verbände etc. und eines möglicherweise damit verbundenen erweiterten Angebotes, beispielsweise mit Internat oder Tagesbetreuung; 4) die sich unterscheidenden Mentalitätskontexte kirchlicher Schulen, die sich auf Aufsichtsintensität und Kontrolldichte staatlicher Behörden auswirken können (landläufig gilt das Klima im Süden der Republik als kirchenfreundlicher als im Norden); 5) vielfältige theologische und spirituelle Motivationsfaktoren unterschiedlicher Schulträger, die sich im Schulalltag ganz praktisch auswirken können (z.B. auch im Blick auf die Zusammenstellung eines nicht bloß 
einheitlichen, sondern uniformen, in sich abgeschlossenen Lehrkörpers);6) die Vielfalt von Schularten, die unterschiedliche Alters- und Gesellschaftsgruppen der Schülerklientel ansprechen (und damit zu großen Unterschieden führen können, was beispielsweise das Alter, die Selbständigkeit, den familiären Rückhalt, die Resilienz der jeweiligen Schüler:innenklientel angeht).

Eine angemessene Historiographie des Missbrauchs an katholischen Schulen hat all diese und noch mehr Faktoren herauszuarbeiten, ihren Wechselwirkungen nachzugehen und einen Beitrag zur Klärung dessen zu leisten, was bei allen Unterschieden vielleicht dennoch plausibilisiert allgemein über den Konnex von katholischen Schulen und Missbrauch ausgesagt werden kann.

Eine derartige historiographische Anstrengung steht bis jetzt noch aus und kann auch im Rahmen der vorliegenden Überlegungen nicht geleistet werden. Dennoch bleibt es im Blick nach vorne unabdingbar, sich den damit verbundenen Mühen zu unterziehen. Die Glaubwürdigkeit und die Verlässlichkeit des Angebots kirchlicher Schulen hängen davon ab. Ohne - auch historiographische - Aufklärung darüber, was missbrauchsbedingende Faktoren sind, bleibt auch an katholischen Schulen die Frage nach der Sicherheit von Kindern und Jugendlichen offen.

Im Sinne eines anfänglichen Versuchs einer Skizze für die notwendige historiographische Aufarbeitung eines möglichen Zusammenhangs zwischen spezifischen Faktoren katholischen Schulwesens und Missbrauch sollen blitzlichtartig im Sinne von Indizien auffällige historisch verbürgte Situationen katholischen Schulwesens in den Blick genommen werden, um denkbare weiterführende historiographische Fragestellungen für weitergehende Forschungen identifizieren und benennen zu können.

Zuvor seien jedoch noch zwei Vorbemerkungen erlaubt: Erstens: Eine historiographische Untersuchung von Missbrauch an katholischen Schulen ist ein wichtiger Beitrag im Sinne der schon vor 40 Jahren vom Erziehungswissenschaftler Wolfgang Brezinka im Blick auf das Schulwesen allgemein geforderten „Geschichte des pädagogischen Aberglaubens".1 Es geht dabei

1 Vgl. hierzu Wolfgang Brezinka, Metatheorie der Erziehung. Eine Einführung in die Grundlagen der Erziehungswissenschaft, der Philosophie der Erziehung und der Praktischen Pädagogik. 4. Aufl. München 1978, 186f. Dort schreibt er: „Die Abkehr von bloßer Geschichtsschreibung der Rechtsgrundlagen, der Schulorganisationsformen, der Schulbesuchsziffern und der verklärenden Schulprogramme hat gerade erst begonnen. Die ,Entmythologisierung der Schulgeschichtsschreibung، erfolgt durch Fragen nach den politischen Interessen der Schulpolitiker, nach den Standesinteressen der Lehrer, nach der Verwirklichung der verkündeten Ideale in der Unterrichtspraxis, nach dem Eigenleben der bürokratischen Schulorganisation (losgelöst von den offiziell für sie genannten Zwecken), nach der Verträglichkeit 
darum, systemische Selbsttäuschungen, Irrtümer und Fehler bezüglich der eigenen Wirksamkeit und Qualität zu thematisieren und aufzudecken. Das nimmt nichts von der Bedeutung katholischer Schulen. Sie bleiben trotz einer solchen "Geschichte pädagogischen Aberglaubens" wichtig. Sie sind wichtig für diejenigen, die sie besuchen, weil sie ein ganzheitliches sinn- und werteorientiertes Bildungsangebot bieten. Sie sind wichtig für die Kirche, weil sie damit Menschen erreichen und in der Folge für ihre Ziele und Überzeugungen gewinnen kann. Sie sind wichtig für die Gesellschaft, weil sie in den unterschiedlichen Schularten einen wesentlichen Teil des Privatschulwesens ausmachen, das zur Pluralisierung des staatlichen Schulwesens beiträgt und damit einem wesentlichen gesellschaftspolitischen Anliegen der verfassungsrechtlichen Gründerinnen und Gründer der Bundesrepublik Deutschland entsprechen. Genau genommen wird diese Bedeutung der katholischen Schulen nicht trotz der infrage stehenden Geschichtsschreibung erhalten bleiben, sondern gerade auch wegen ihr. Im Grunde ist sie ein Beitrag zu den vielfältigen Qualitätssicherungsmaßnahmen, denen sich das katholische Schulwesen schon seit geraumer Zeit unterzieht. ${ }^{2}$ Zweitens: Eine historiographische Untersuchung von Missbrauch an katholischen Schulen baut auf einer - wie weiter oben schon angedeutet - multifaktoriellen Herangehensweise auf und berücksichtigt die Eigenart des Untersuchungsgegenstandes „System Schule“. Gerade mit Letzterem soll gewährleistet werden, dass zentrale systemische Aspekte ausreichend wahrgenommen, angemessen gewichtet und in ihren spezifischen Wechselwirkungen berücksichtigt werden. Als einige Kernelemente des Systems Schule sollen nachfolgend diese Punkte anfanghaft beleuchtet werden: in personaler Hinsicht die Lehrer und das Lehrerkollegium, in struktureller Hinsicht die Schulorganisation und die Schulpolitik, in ideeller

der vielen gleichzeitig verfolgten Erziehungsziele, nach den Unterschieden zwischen dem Selbstbild der Lehrer und dem Lehrerbild der Schüler, dem Selbstbild der Schüler und dem Schülerbild der Lehrer, nach dem Unterrichtserfolg in allen Fächern und den Ursachen der Mißerfolge, nach den ungewollten Nebenwirkungen wie schulbedingte psychische Erkrankungen von Schülern und Lehrern, Schulverdrossenheit von Schülern und Eltern, unerwartete Einstellungsänderungen großer Absolventengruppen, schullaufbahnbedingte Arbeitslosigkeit usw. In einer Gesellschaft, die häufig ,Erziehungsgesellschaft' genannt, in der an die Macht der Erziehung geglaubt und von den Nutznießern dieses Glaubens die ,permanente Erziehung‘ gefordert wird, bestünde der größte Dienst, den die Erziehungshistoriker ihr leisten könnten, vielleicht darin, ,eine Geschichte des pädagogischen Aberglaubens' zu schreiben."

2 Vgl. hierzu z.B. die Darstellung „Profil und Qualität“ auf dem Internetportal der Katholischen Schulen, einzusehen unter https://schulen.katholisch.de/Profil-und-Qualität, zuletzt aufgerufen am 29.9.2020. 
Hinsicht das Bild vom Kind und die Kindheit, in prozessualer Hinsicht die Pädagogik und das pädagogisch motivierte Handeln.

\section{Lehrer und Lehrerkollegium}

Lehrer und Lehrerinnen, vor allem auch an kirchlichen Schulen, sind Idealisten, so zumindest die landläufige Vorstellung. Sie sind dies, weil sie sich bewusst entschieden haben, sich bei einem kirchlichen Arbeitgeber zu verpflichten. Sie müssen eine idealistische Ader haben, weil sie aus dem Glauben mit der Kirche für ein Werte- und Sinnsystem offenbar in besonderer Weise einstehen wollen und nicht „nur“ einen Job machen wollen. Dabei nehmen sie anscheinend mehr oder weniger finanzielle Nachteile in Kauf, die sich für sie als Angestellte gegenüber den verbeamteten Lehrerinnen und Lehrern im staatlichen Schulsystem ergeben. Sie gehen an kirchliche Schulen, obwohl diese vielleicht schlechter ausgestattet sind als staatliche Schulen, weil sie sich bei „Kirchens" ein besseres Betriebsklima und vielleicht sogar mehr kreative Spielräume im Blick auf Unterricht sowie Schulleben erhoffen und sich dabei einbringen möchten.

Idealisten und Idealistinnen haben jedoch immer wieder ein großes Problem. Es sind zu wenige, um damit all die Lehrerstellen zu besetzen, die Kirche zu bieten hat. So kommt es schnell zu dem, wozu es nahezu unausweichlich kommen muss, nämlich einem signifikanten Personalmangel, mit dem es aktiv umzugehen gilt. Grundsätzlich kann es da grob gesprochen zwei Richtungen geben. Die eine davon zielt auf Bestenauslese ab, möchte jene Lehrkräfte gewinnen und binden, die sich durch ihre Persönlichkeit und ihr fachliches Können auszeichnen. Wer solche Kräfte gewinnen will, der muss auch etwas zu bieten haben, jedenfalls mehr als fromme Sprüche und farbenfrohes kirchliches Brauchtum im Schulalltag. Konkret bedarf es da der Beschäftigung z.B. mit den Fragen nach einer angemessenen Bezahlung, nach der flexiblen Gestaltung von (Lebens-)Arbeitszeiten, nach Unterstützungssystemen, nach Entwicklungs- und Weiterqualifizierungsmöglichkeiten oder nach arbeitsvertraglichen Regelungen, die nicht durch unangemessene Loyalitätsanforderungen bis in die persönlichsten Lebensbereiche hinein belastend wirken. Die zweite Richtung, die man einschlagen kann, um sein Personalmangelproblem zu lösen, setzt eher auf die Reduzierung der Anforderungen an das zukünftige Personal. Die Quantität mag dann noch stimmen, aber ob dies dann auch noch für die Qualität gilt? Weniger gute Abschlussnoten, weniger Hinweise auf die Bereitschaft zum Engagement und auf Kreativität, 
schwächere Identifikation mit dem beruflichen Aufgabenfeld, weniger Beachtung von Eigenheiten und vielleicht sogar leichten Auffälligkeiten mögen zwar dazu beitragen, den Lehrkörper zahlenmäßig auf dem passenden Niveau zu halten, aber ob man damit allen Beteiligten einen Gefallen tut? Bis zu einem gewissen Maß mag eine solche Herangehensweise hilfreich sein, schlussendlich muss jedes Team auch mit einem gewissen Anteil schwächerer Mitglieder umgehen können. Nichtsdestotrotz kann es aber eine kritische Grenze geben, ab der Unterschiede nicht mehr kompensiert, sondern nur noch verstärkt werden. Die Schwächeren innerhalb eines Lehrerkollegiums können sich dann schneller überfordert, isoliert, übergangen fühlen, fallen vielleicht leichter in ein Burnout, eine Verweigerungshaltung, emigrieren innerlich und suchen gar Nähe und Unterstützung bei denen, die eigentlich ihre Schutzbefohlenen sind. Fachliche Qualifikation und persönliche Eignung der Lehrkräfte bleiben nicht ohne Auswirkung auf die Beziehungsgestaltung zu den Schülern. Im schlechten Fall haben die Schüler und Schülerinnen darunter zu leiden und es kann sich im schlechtesten Fall unter Umständen in missbräuchlichen Beziehungsstrukturen abbilden.

Schwierigkeiten im und mit dem Lehrkörper an katholischen Schulen ergeben sich aber nicht nur aus einem unangemessenen Umgang mit Personalmangel, sondern auch auf Grund quasi "kirchenpolitischer" Vorgaben. Was ist z.B. mit der Ordensschwester, die von ihrer Gemeinschaft mehr oder weniger zwangsweise für den Schuldienst in der ordenseigenen Schule „abkommandiert" wird, um so angeblich deren Profil zu gewährleisten, obwohl sie keinen Zugang zu Kindern und zu den Anforderungen des Berufsfeldes hat? Was ist mit dem Schulleiter, der die notwendigen fachlichen Qualifikationen gerade noch so erfüllt, aber deshalb die Stelle erhalten hat, weil er sich in Moral- und Glaubensdingen als vollidentifiziert mit dem Lehramt der Kirche gibt? Was ist mit den Religionslehrerinnen, den Religionslehrern oder den Priestern, die sich nicht an didaktischen Standards, an Qualitätssicherungsmaßnahmen etc. des Kollegiums beteiligen, weil sie sich im Kontext einer kirchlichen Schule als so relevant erachten, dass sie meinen sich erlauben zu können, auf fachliche und persönliche (Weiter-)Bildung zu verzichten?

Vor diesem knapp skizzierten Hintergrund wäre es aus dem Blickwinkel einer kritisch-produktiven Schulgeschichtsschreibung z.B. interessant zu wissen:

- Nach welchen Kriterienkatalogen wurde und wird Personal an kirchlichen Schulen ausgesucht?

- Welche Schwerpunkte wurden in diesen Katalogen im Laufe der Zeit gesetzt und wie haben sie sich verändert? 
- Auf welche Haltungen wurde bzw. wird bei der Lehrerschaft das Augenmerk gelegt und wie wird versucht, hier Einfluss zu nehmen? War vielleicht das Thema Fügsamkeit und Kirchlichkeit wichtiger als z.B. die Identifikation mit den Nöten und Sorgen der Schülerinnen und Schüler oder Eltern? ${ }^{3}$

- Wie hat sich die Vertretung berufsständischer Anliegen gegenüber dem Arbeitgeber Kirche entwickelt und welche Auswirkungen hat dies auf die Qualifikation sowie die Motivation der Lehrkräfte?

- Auf welche Weise haben sich die Unterstützungssysteme für Lehrkräfte an kirchlichen Schulen im Blick auf die Förderung der fachlichen und persönlichen Qualifikation entwickelt, ausgestaltet und ausgewirkt? Und gibt es solche Unterstützungssysteme schon immer in ausreichendem Maß, so dass auch Teamfähigkeit und kollegiale Beratung/Unterstützung gepflegt werden? Dies ist übrigens ein wichtiger Gesichtspunkt, wenn es um die Meldung von missbräuchlichen Geschehen aus dem bzw. um Aufarbeitung dieser mit dem Kollegium gehen soll.

- Wie haben sich - relativ gesehen - die materiellen Aufwendungen von Kirche für die von ihr getragenen Schulen bzw. das dort für sie tätige Personal entwickelt? Das Aufgabenportfolio der Kirche beschränkt sich nicht auf Schulen. Inwiefern veränderte sich im Laufe der Zeit die Aufmerksamkeit und das Ausmaß der Fürsorge für das kirchliche Schulwesen im Vergleich $\mathrm{zu}$ anderen kirchlichen Bereichen?

\section{Schulorganisation und Schulpolitik}

Man muss es nicht gleich so sehen, wie es im folgenden Zitat zum Ausdruck kommt:

"[... die Schulaufsicht durch Geistliche ist sittlich verwerflich, also unsittlich, weil sie in der Gegenwart sich wider den Sinn der Schulwohlfahrt und der vollkommenen Lebensgestaltung des Lehrerstandes wendet, weil ihre Unfähigkeit in der wissenschaftlichen Führung und ihr Charakter als Polizeikontrolle hemmend und schädigend wirkt, weil sie einem anderen Stande die Berufsehre nimmt, ihn dauernd niederdrücken und in Knechtsbanden halten will, weil sie also einen Angriff auf das Wohl anderer darstellt, sei es der Lehrerstand oder

3 Zum Thema Haltung und Lehrer vgl. den Sammelband von Carolin Rotter/Carsten Schülke/ Christoph Bressler (Hrsg.), Lehrerhandeln - eine Frage der Haltung? Weinheim/Basel 2019. 
alle in der Volksschule Gebildeten, weil sie nicht im Sinne der vollen Lebensbereicherung wirkt."4

Harte Worte. Sie stammen aus dem Jahr 1919, als nach dem Untergang der alten monarchischen Ordnung die sogenannte geistliche Schulaufsicht abgeschafft wurde. Es handelte sich dabei um eine durch den Staat den Geistlichen zugewiesene Aufsicht über das jeweilige (wohlgemerkt: staatliche) Volksschulwesen vor Ort, wobei diesen weitgehende Rechte zur Beurteilung und Disziplinierung von Lehrkräften im Blick auf deren fachliche, persönliche und moralische Eignung eingeräumt wurde. Auch wenn es diese geistliche Schulaufsicht nun seit 100 Jahren nicht mehr gibt und der Staat alle Schulen, auch die des Privatschulwesens, zu dem die katholischen Schulen zählen, seinem Hoheitshandeln klar unterstellt hat, gibt es doch gewisse Anstöße, den Blick in die jüngere Zeit zu wenden. Bis in unsere Tage war und ist es weitgehend üblich, dass die Schulabteilungen der Ordinariate in den (Erz-)Diözesen von Geistlichen geleitet wurden, die dem Domkapitel angehören. Da mit der Zugehörigkeit zu einem Domkapitel alleine bekanntermaßen nicht unbedingt automatisch eine einschlägige und grundständige Ausbildung in Schulpädagogik oder Erziehungswissenschaften verbunden ist, stellt sich schon die Frage, mit welchen Kompetenzen und Motivationslagen jene Abteilungen geleitet wurden. Es lässt sich zumindest nicht automatisch von der Hand weisen, dass hier unter Umständen Fachfremdes und Kirchenpolitisches eher zum Tragen gekommen sein könnten als der feste Wille und das Vermögen zu eigenständigen fachlichen Beiträgen zum Wohl der kirchlichen Schulen und der an ihnen lebenden und lernenden Menschen. Damit soll keinesfalls gesagt sein, dass hier nicht auch viel Gutes geschehen ist. Dennoch macht es wahrscheinlich beispielsweise einen Unterschied, ob die Meldung von Missbrauchsvorwürfen an einer katholischen Schule bei den Letztverantwortlichen mehr von kirchenpolitischen Erwägungen her wahrgenommen wird oder mit dem geschulten Blick eines Fachmanns bzw. einer Fachfrau, der/die qualifiziert abschätzen kann, was Missbrauch für den konkret Betroffenen bedeutet.

Wie leicht Politisches über Pädagogisches die Oberhand gewinnen kann, zeigt ein kurzer Blick in die Geschichte. Es ist ca. 100 Jahre her, dass es in einem Lexikon für Pädagogik hieß:

4 Jacob Beyhl (Hrsg.), Die geistliche Schulaufsicht eine unsittliche Einrichtung. Eine Enthüllung klerikal-reaktionärer Treibereien. Amtliche Urkunden zu meiner letzten Maßregelung. München 1919, 33 f. 
„Die in den Worten: ,Die Kirche den Theologen, die Schule den Pädagogen', sich aussprechende Auffassung von der Stellung der Volksschule und des Lehrerstandes verkennt die Aufgabe und das Wesen der Volksschule auch die gerechten Ansprüche des Berufes an die Selbständigkeit in der Schularbeit. Die Schule ist im Kulturleben dienender Faktor, sie ist Hilfsanstalt für Familie, Kirche und Staat und nicht wie diese eine ursprüngliche gesellschaftliche Form; deshalb muss sie sich auch in ihrer Wirksamkeit der Abhängigkeit von den genannten Faktoren bewusst bleiben. ${ }^{5}$

Dass die Kirche in Bezug auf das Schulwesen, die Gesellschaft, die Schüler und Schülerinnen dienende Funktion hat und sich daher auf die Eigengesetzlichkeiten des Schulwesens einzulassen hat, scheint hier in weiter Ferne zu liegen. Eine gewisse Verstärkung dieses Eindrucks kann man bei den Auseinandersetzungen um die Abschaffung der sogenannten Konfessionsschulen in der Mitte des vergangenen Jahrhunderts gewinnen. Wohl aus Furcht, in kirchenpolitischer Hinsicht gesellschaftlichen Einfluss zu verlieren, haben manche Kirchenvertreter lange Zeit das Prinzip verteidigt, Kinder entsprechend der Konfessionszugehörigkeit im staatlichen Volksschulsystem in unterschiedlichen, je nach Konfession getrennten Schulen zu separieren. Dass dies zu erheblichen Nachteilen für die Kinder aus pädagogischer Sicht führte, spielte anscheinend keine große Rolle. Die Konfessionalisierung des Schulsystems hat vor allem auf dem Lande mit dazu beigetragen, dass zahlreiche, wegen ihrer geringen Schülerzahl und mangelnden Gliederung sogenannte „Zwergschulen“ Orte schulischer Bildung mit mangelhafter Ausstattung und entsprechend qualifizierter Lehrerschaft waren. ${ }^{6}$

Im Zusammenhang mit diesen, zwar für das Verhältnis der Kirche zum staatlichen Schulsystem feststellbaren Beobachtungen, deren Relevanz sich für das Verhältnis der Kirche zu ihren eigenen Schulen nicht im Vorhinein ausschließen lässt, wäre es interessant, zur weiteren Klärung auch den folgenden und weiteren damit verbundenen Fragen schulhistoriographisch nachzugehen: - Wie hat sich die kirchliche Governance ${ }^{7}$ bezüglich Schulen in kirchlicher Trägerschaft entwickelt? Welche Gesichtspunkte haben dabei eine Rolle gespielt?

5 J.J. Wolff, Artikel: Lehrer an Volksschulen, in: Lexikon der Pädagogik, Bd. 3. Freiburg i. Breisgau 1921, 230-234, hier 232.

6 Vgl. Albert Reble, Das Schulwesen, in: Max Spindler (Hrsg.), Handbuch der Bayerischen Geschichte, Bd. 4: Das neue Bayern 180o-1970, 2. Teilband. München 1975, 949-99o, hier v.a. $986 f$.

7 Christiane Frantz/Hanna Grotz, Identität katholischer Schulen gestalten. Die Analyse von Normenprozessen und Rollen aus der Perspektive politikwissenschaftlicher 
- Welche Ausformungen haben das Zueinander von kirchlicher und staatlicher Governance im Laufe der Zeit angenommen? Ist eine Phase der Abgrenzung durch jene einer gewissen Gleichgültigkeit abgelöst worden, in der die Vertreter staatlicher Governance jene der kirchlichen Governance grundsätzlich einfach gewähren lassen?

- Wer hat die Governance kirchlicher Schulen übernommen und wie waren diese Personen dafür qualifiziert bzw. darauf vorbereitet?

- Wie hat sich das Verständnis von Governance in Kirche allgemein, welches u.a. aus theologischen Gründen stark von hierarchischem Denken geprägt war und ist, auf dem pädagogischen Feld ausgewirkt?

- Gibt es im Laufe der Zeit eine Entwicklung von Qualitätssicherungsinstrumenten der kirchlich zu verantwortenden Governance? Gibt es dauerhafte Unterschiede zum staatlichen Bereich und/oder gibt es gemeinsam aufeinander bezogene?

\section{Bild vom Kind und von Kindheit}

Kinder und Kindheit stehen immer wieder auf unterschiedlichste Weise im Fokus der Aufmerksamkeit. Sei es wegen einer mehr oder weniger unmittelbaren Betroffenheit, wenn es um den Lärm im Umfeld einer Kindertagesstätte geht, sei es im politischen Diskurs z.B. über Migration und deren Folgen, sei es im wissenschaftlichen Diskurs wie der Kindheitsforschung als Versuch der Ergründung der Möglichkeit gelingenden Aufwachsens, sei es sogar in dem Bestreben, sich als Gesellschaft besser selbst zu verstehen. Nicht umsonst trägt einer der letzten Ausgaben der wohl mit bekanntesten Kultur- und Politikzeitschrift Kursbuch den Titel „Menschenskinder“. Es soll dabei um die Bedeutung von Kindern für die Gesellschaft gehen. Einer der Autoren in diesem Heft, Armin Nassehi, macht dabei die Unterscheidung zwischen kindlich und kindisch, wobei Letzteres die Zeitsignatur unserer gegenwärtigen Gesellschaft sei. Er sagt ernüchternd: „Die kindische Gesellschaft [...] kennt nur die Unmittelbarkeit - ihr ist letztlich die grundlegende kindliche Fähigkeit fremd: die temporalisierte Entwicklungsfähigkeit.“8 Kann man so sehen, muss man aber nicht. Eines hingegen muss man schon. Kind und Kindheit haben nicht nur in der Soziologie paradigmatischen Charakter, sondern auch und

Governance-Konzepte, in: Judith Könemann/Denise Spiekermann (Hrsg.), Katholische Schulen - Herausgeforderte Identität. Paderborn 2019, 61-87.

8 Armin Nassehi, Warum die Gesellschaft kindisch ist. Eine Ehrenrettung des Kindlichen, in: Kursbuch 201, 2020, 161-178, hier 177 . 
gerade in der Pädagogik. Nicht umsonst heißt es: „Kindheitskonstruktionen sind die Voraussetzungen pädagogischer Theorien und erfüllen dort häufig die Funktion einer Letztbegründung pädagogischen Handelns.“9 Das, was man unter „Kind“ bzw. „Kindheit" versteht, hat Einfluss darauf, welche Ziele man mit Bildung und Erziehung verfolgen will, welche Mittel und Maßnahmen man für geeignet hält, um Kinder in die durch die Ziele gesetzte Richtung mit der Zeit zu bewegen, welches Verhalten als "normal“ oder "auffällig“ und daher behandlungsbedürftig erscheint. ${ }^{10}$ Wenn gerade vorhin das Wort „Kindheitskonstruktionen" Verwendung fand, so weist dies auf eine grundlegende Schwierigkeit hin: Das Kind oder die Kindheit als solches gibt es kaum. Vieles hängt davon ab, welche Prämissen in Anschlag gebracht, welche Vorverständnisse wirksam und welche Interessen auch immer (mehr oder weniger ausdrücklich formuliert) verfolgt werden. Es verwundert daher kaum, wenn im Laufe der Geschichte unterschiedliche Verständnisse dessen, was Kindsein und Kindheit bedeutet, oder zumindest bedeuten könnte, auszumachen sind.

Im Blick auf das Bild vom Kind im kirchlichen Kontext hat Anton A. Bucher eher Beunruhigendes herausgearbeitet. So habe die Kirche über lange Zeit das Bild vom Kind im Rückgriff auf religiöse Denkfiguren folgendermaßen gezeichnet:

„Das Bild des Jesusknaben, der geradezu beunruhigend artig ist, nicht in die Pubertät kommt und permanent im Gesichtsfeld der Mutter verbleibt [...]. Das Bild von Gott Vater, der seine Kinder nicht heranwachsen lässt. Sei es, indem er ihnen vorrechnet, was er [...] alles für Sie getan habe, so dass sie genötigt werden, diese unsägliche Schuld mit kindlicher Dankbarkeit abzustottern. Sei es indem er mit ihnen verfährt, wie er will, sodass sie keinen Eigen-Sinn und keinen eigenen Willen ausbilden können, sondern knechtisch gehorchen. Das Bild von Maria, die in geschlechtlicher Hinsicht Kind bleibt [...] aber als ,Mutter' ihren Mantel über Erwachsene breitet, sofern sie demütig und brav genug waren. Das Bild von Mutter Kirche, die (...) von ihren Kindern vor allem Loyalität erwartet.11

Wenn man sich Beschreibungen von Täter-Opfer Konstellationen hinsichtlich der Missbrauchsfälle im Verantwortungsbereich der Kirche ins Gedächtnis ruft, dann dürfte ein Bild vom Kind wie eben skizziert mehr als beunruhigen.

$9 \quad$ Gerold Scholz, Die Konstruktion des Kindes. Über Kinder und Kindheit. Opladen 1994, 9.

10 Interessante Beispiele liefert in diesem Zusammenhang für den vorschulischen Bereich die Arbeit von Alex Knoll, Kindheit herstellen. Diskurs, Macht und soziale Ungleichheit in Betreuung und Alltagsgestaltung. Wiesbaden 2016.

11 Anton A. Bucher, Braucht Mutter Kirche brave Kinder? Religiöse Reifung contra kirchliche Infantilisierung. München 1997, 205f. 
Es liegt der Verdacht nahe, hier bestehen wirksame Bezüge und Wechselwirkungen. So gesehen wären u.a. wichtige Forschungsfragen:

- Welche Bilder vom Kind bzw. der Kindheit gibt es in der Tradition der Kirche und welche wurden wie im schulischen Kontext relevant?

- Wie unterscheiden sich im Laufe der Zeit die Bilder vom Kind bzw. der Kindheit in offiziellen Kirchendokumenten von denjenigen in der pädagogischen Arbeit vor Ort?

- Inwiefern unterscheiden sich die Verständnisse von Kindheit und Kind innerhalb des Verantwortungsbereichs der Kirche von denjenigen außerhalb der Kirche und inwiefern gibt es Wechselwirkungen?

\section{Pädagogik und pädagogisch motiviertes Handeln}

Ein wesentlicher Baustein im Kampf gegen den Missbrauch von Kindern und Jugendlichen ist neben der ausdrücklichen Auseinandersetzung mit der Thematik Missbrauch eine vom grundsätzlichen Gedanken der Resilienz geprägte Erziehung und Bildung. Präventiv heißt dies generell, die Widerstandskräfte und Bewältigungsstrategien von Kindern und Jugendlichen zu stärken, wenn nötig grundzulegen und wenn möglich auszubauen. Dies kann nur gelingen, wenn Kinder und Jugendliche im Mittelpunkt schulischen Lebens stehen. Dabei geht es nicht nur darum, sich entsprechendes Wissen, wie z.B. das um die eigenen Rechte, aneignen zu können, also mehr oder weniger theoretisch Bescheid zu wissen. Es geht auch darum, dass Kinder und Jugendliche in der Art und Weise, wie unterrichtet wird und wie der Schulalltag gelebt wird, ganzheitlich erfahren, was es heißt, Würde zu haben, Grenzen zu respektieren, wertschätzend miteinander umzugehen, Gerechtigkeit zu erfahren, Solidarität zu üben, Verantwortung zu übernehmen etc. Hierzu braucht es ein ganzheitliches pädagogisches Konzept, das berücksichtigt, dass Schule weniger ein funktionales Betriebsgebäude mit effektiven Produktionsmethoden darstellt, sondern vielmehr ein komplexes Lern-, Entwicklungsund Lebensgefüge.

Trotz mannigfacher Bemühungen, dementsprechende pädagogische Ansätze in katholischen Schulen wirksam werden zulassen - man denke hier nur an zahlreiche reformpädagogisch motivierte Initiativen und Maßnahmen $^{12}$ - haben katholische Schulen mit Gegebenheiten umzugehen, die

12 Vgl. hierzu z.B. Margret Rasfeld, Reformpädagogik in katholischen und evangelischen Schulen, in: Heiner Barz (Hrsg.), Handbuch Bildungsreform und Reformpädagogik. Wiesbaden 2018, 287-300. 
nicht zwangsläufig, aber leicht gegenteilig wirksam werden können. Nicht ganz von der Hand zu weisen ist, dass katholische Schulen manchmal als angenehmere Alternative zu staatlichen Schulen wahrgenommen werden, teilweise im Vergleich dazu sogar in gewissem Sinne als Eliteschulen oder zumindest doch als Schulen von und für einen wie auch immer gearteten speziellen Kreis von Adressaten. Dies kann zu Abgrenzungstendenzen und starken Bindungskräften nach innen führen, was Kritik und (Selbst-) Kritikfähigkeit unter Umständen erschwert. Solche internen Kohäsionskräfte werden zum Teil auch durch die Verbindung der katholischen Schule mit einem Internat verstärkt. Die gemeinsame Zeit und damit auch das wechselseitig Aufeinander-verwiesen-Sein sind damit zwangsläufig intensiver. Herausforderungen für ein katholisches Schulwesen, das sich um ein resilienzrelevantes pädagogisches Konzept bemüht, dürften auch das stark hierarchisch geprägte kirchliche Umfeld und die damit verbundenen Mentalitäten sein. Hier das Konzept von Eigenständigkeit, Selbstbewusstsein und Mündigkeit zu fördern ist möglich, genauso wie die Tatsache, dass es diesbezüglich zu Reibungen kommen kann. Pluralität, Diversität im Blick auf alle Beteiligten und deren Überzeugungen, deren freies, kritisches Denken bedürfen hier so gesehen schon einer besonderen Fürsorge. Dies gilt umso mehr, wenn man bedenkt, dass sich Kirche als Sendungsgemeinschaft versteht, die an die Offenbarung Gottes gebunden ist.

Vor diesem speziellen Hintergrund gilt es historiographisch folgenden Fragen nachzugehen:

- Welche pädagogischen Grundlinien zeigen sich als prägend für katholische Schulen im Laufe der Zeit?

- Welches Schulklima herrschte und herrscht an katholischen Schulen? Oder konkret gefragt: Wie wird das pädagogische Konzept im Alltag erfahrbar?

- Inwiefern zeigt sich im Zeitlauf das pädagogische Konzept als wirklich ganzheitlich, indem es Familien und Eltern miteinbezieht? Oder auch hier anders gefragt: Wie weit ausgreifend entwickelten sich katholische Schulkonzepte?

\section{Vergangenheit, Gegenwart und Zukunft}

Ein historiographischer Blick auf kirchliche Schulen im Sinne einer Geschichte pädagogischen Aberglaubens, in der versteckt wirkende oder offen sich zeigende Mechanismen und Strukturen als Ursache für Spannungen zwischen Schein und Sein, Idee und Realität herausgearbeitet werden, kommt nicht ohne den Blick auf die Schullandschaft sowie die Gesamtgesellschaft aus. Zu 
groß sind die Wechselwirkungen und wechselseitigen Verstrickungen. Aus der Geschichte kirchlicher Schulen lässt sich einiges über die nichtkirchlichen Schulen sowie deren gesellschaftliches Umfeld lernen. Umgekehrt gilt das genauso. Positionen und Positionierungen im Kontext kirchlicher Schulen haben sich oft durch (teilweise) rigorose Abgrenzung aber auch bewusste Übernahme oder unreflektierte Nachahmung herausgebildet. ${ }^{13}$

Grundsätzlich lässt sich von daher nochmals resümierend sagen:

Erstens: Eine solche Geschichte kann nicht anders als multiperspektivisch und interdisziplinär angegangen werden.

Zweitens: Eine solche Geschichte ist offen und fluide sowohl in Bezug auf die Perspektive, von der her sie geschrieben wird als auch auf den jeweiligen inhaltlichen Fokus hin, auf den besonderes Gewicht gelegt wird.

Drittens: Eine solche Geschichte kann uns keine vollständige Sicherheit bezüglich eines Erkenntnisfortschritts in Richtung der Idee von der guten (kirchlichen) Schule geben. Sie kann uns aber eindeutig verdeutlichen, wie wichtig es ist, das jeweilige Handeln auf seine Einflussfaktoren, Mechanismen und Zusammenhänge selbst zu hinterfragen.

Kurz gesagt: Ein kritischer historiographischer Blick auf kirchliche Schulen lehrt Demut, trotz aller Notwendigkeit selbstbewusst auf einem freien Schulmarkt zu bestehen, trotz allem Idealismus und trotz beeindruckender Erfolge.

In diesem Zusammenhang lässt sich dann auch in angemessen differenzierter Weise klären, ob es so etwas wie einen "katholischen Geschmack“ (Klaus Mertes) oder präziser gesagt ein katholisches Spezifikum von Missbrauch an katholischen Schulen gibt. Bisher lässt sich dies meiner Einschätzung nach zumal aus historiographischer Sicht im weiter oben dargelegten Sinne - mit den vorliegenden Studien nicht mit hin- und ausreichender Sicherheit sagen. ${ }^{14}$ Handelt es sich um einen "katholischen“ Missbrauch insofern er an

13 Interessant dürfte in diesem Kontext der Versuch einer historisch motivierten Betrachtung des Faches Religionspädagogik und dessen Abhängigkeit von den jeweiligen Zeitbedingungen sein. Vgl. hierzu Friedrich Schweitzer/Henrik Simojoki, Moderne Religionspädagogik. Ihre Entwicklung und Identität. Gütersloh 2005, hier bes. 27-222. Wenn sich schon dieses Fach, in dem es eigentlich um den entscheidenden Punkt kirchlicher Verkündigung geht, als so zeitbedingt zeigt, wie muss dies dann erst recht für das Gesamtsystem Schule gelten? Immerhin müsste sich doch gerade im Bereich Religionspädagogik das zeigen, was das Alleinstellungsmerkmal kirchlichen Handelns ausmacht: widerständig prophetisch zeigen, was die Kirche zum Salz der Erde macht.

14 Vgl. hierzu z.B. Heiner Keupp u.a. (Hrsg.), Sexueller Missbrauch, psychische und körperliche Gewalt im Internat der Benediktinerabtei Ettal. Individuelle Folgen und organisatorischstrukturelle Hintergründe, München 2013, einzusehen unter www.uuuuuuuuu.de/ dokumente/130307_ipp_Studie_Ettal.pdf, zuletzt aufgerufen am 26.4.2021; Ursula Raue, Bericht über Grenzverletzungen gegenüber Kindern und Jugendlichen im Jesuiten-Kolleg 
katholischen Schulen stattfindet oder gibt es an katholischen Schulen Missbrauch, weil es dort spezifische Faktoren gibt, die Missbrauch begünstigen und nur an katholischen Schulen vorkommen? Bisher zeigen sich im Wesentlichen in unterschiedlichen Schattierungen "nur" solche missbrauchsbegünstigenden Faktoren, die es in kirchlichen Schulen genauso wie in nichtkirchlichen Schulen geben kann: ungute Abschottung (z.B. durch das Selbstverständnis Eliteschule oder zumindest etwas Besonderes zu sein) unklare Rollenverständnisse und Machtverhältnisse (z.B. Lehrer:innen, die sich Guru-ähnlich inszenieren) Absolutheitsansprüche (beispielsweise in pädagogischen Konzepten) etc. ${ }^{15}$ Die Wirksamkeit dieser Faktoren ist nahezu gleich, auch wenn sie sich hinsichtlich der Herleitungen ihrer Existenz unterscheiden mögen. Erwachsen sie nun aus einem kirchlich-theologischen oder eher säkularen Kontext, in ihrem Entstehungszusammenhang und ihrer Wirkungsfolge stellen sie auf jeden Fall einen verheerenden Irrweg dar.

St. Blasien, 15.2.2011, einzusehen unter www.jesuiten.org/fileadmin/user_upload/Downloads/Bericht_Raue_St._Blasien_-_15.02.11.pdf, zuletzt aufgerufen am 26.4.2021; Julia Zinsmeister/Petra Ladenburger/Inge Mitlacher, Schwere Grenzverletzungen zum Nachteil von Kindern und Jugendlichen im Aloisiuskolleg Bonn - Bad Godesberg. Abschlussbericht zur Untersuchung im Auftrag der Deutschen Provinz der Jesuiten, 2011, einzusehen unter www.jesuiten.org/fileadmin/user_upload/Downloads/Abschlussbericht_AKO_Zinsmeister.pdf, zuletzt aufgerufen am 26.4.2021. Im Konnex zu diesen Untersuchungen für das kirchliche Umfeld interessant die Ausführungen zur Odenwaldschule: Claudia Burgsmüller/Brigitte Tilmann, Abschlussbericht über die bisherigen Mitteilungen über sexuelle Ausbeutung von Schülern und Schülerinnen an der Odenwaldschule im Zeitraum 196o bis 2010. Wiesbaden/Darmstadt 2010, einzusehen unter www.anstageslicht. de/fileadmin/user_upload/Geschichten/Missbrauch_-_eine_unendliche_Geschichte_ auch_in_Deutschland/OSO_Abschlussbericht2010.pdf, zuletzt aufgerufen am 26.4.2021.

15 Vgl. hierzu Claudia Bundschuh, Sexualisierte Gewalt gegen Kinder in Institutionen. Nationaler und internationaler Forschungsstand. Expertise im Rahmen des Projekts „Sexuelle Gewalt gegen Mädchen und Jungen in Institutionen“, München 2010, einzusehen unter www.dji.de/fileadmin/user_upload/sgmj/Expertise_Bundschuh_mit_ Datum.pdf, zuletzt aufgerufen am 26.4.2021. In Verbindung mit Deutsches Jugendinstitut e.V. (Hrsg.), Sexuelle Gewalt gegen Mädchen und Jungen in Institutionen. Abschlussbericht des DJI-Projekts „Sexuelle Gewalt gegen Mädchen und Jungen in Institutionen“, München 2011, einzusehen unter www.dji.de/fileadmin/user_upload/sgmj/Abschlussbericht_Sexuelle_Gewalt_02O32012.pdf, zuletzt aufgerufen am 26.4.2021. 